\title{
Sense of agency during and following recovery from anorexia nervosa
}

Manja M. Engel ${ }^{* 1}$, Vivian Ainley, Manos Tsakiris ${ }^{2,3,4}$, H. Chris Dijkermann ${ }^{1}$, Anouk Keizer ${ }^{1}$ ${ }^{1}$ Department of Experimental Psychology, Utrecht University

${ }^{2}$ Department of Psychology, University of London

${ }^{3}$ The Warburg Institute, University of London

${ }^{4}$ Department of Behavioural and Cognitive Sciences, University of Luxembourg

\section{Author Note}

Manja Engel (D) https://orcid.org/0000-0002-5158-6246

Vivien Ainley (iD https://orcid.org/0000-0003-0373-0079

Manos Tsakiris (D) https://orcid.org/0000-0001-7753-7576

H. Chris Dijkerman (iD https://orcid.org/0000-0002-5364-3312

Manja M. Engel is now at the Department of Experimental Psychology, Faculty of Social and Behavioural Sciences, Utrecht University.

We have no known conflict of interest to disclose.

Correspondence concerning this article should be addressed to Manja M. Engel, Utrecht University, Faculty of Social and Behavioural Sciences, Experimental Psychology/Helmholtz Institute, 3584 CS Utrecht, The Netherlands. Email: m.m.engel@uu.nl 


\begin{abstract}
The need to feel in control is central to anorexia nervosa (AN). AN patients tend to use dysfunctional behaviour to feel a sense of control. The sense of control in AN has only been studied through selfreport. The aim of this study is to investigate an implicit sense of control, the sense of agency (SoA), in AN patients, recovered AN (RAN) patients and HC. We hypothesized that AN patients would exhibit a lower SoA compared to RAN patients and HC. Furthermore, we expected that state-anxiety would negatively predict SoA, and that this would be more prominent in AN patients, as studies have shown that SoA can be influenced by negative emotional states. The SoA was measured with the intentional binding task and state-anxiety levels through a questionnaire. Results showed no differences in SoA between groups. Findings did show that AN patients had significantly higher state anxiety scores compared to RAN patients and $\mathrm{HC}$, and RAN patients had higher state anxiety scores compared to $\mathrm{HC}$. However, state anxiety was not a significant predictor of SoA. We did not find any evidence of differences in SoA between groups. Further studies should focus on specific aspects of the need for control in AN by, for example, by manipulating (un)certainty in these paradigms.
\end{abstract}

Keywords: anorexia nervosa, recovered, sense of agency, intentional binding, anxiety 


\section{Sense of agency during and following recovery from anorexia nervosa}

Many people have experienced loss of control, for example, in a relationship or at work, or over their thoughts and emotions. Less common, however, is the experience of losing control over actions such as switching on the light - 'was it me who turned on the light?' The latter refers to a more implicit experience of control that is called sense of agency (SoA) (Haggard, 2017). Several psychiatric groups, such as those with obsessive-compulsive disorder and schizophrenia, struggle with respect to experiencing control as well as SoA (Haggard et al., 2003; Oren et al., 2019). A disorder in which SoA has not been investigated yet, but in which the need for control is a central component, is anorexia nervosa (AN) (Fairburn et al., 2003; Froreich et al., 2016; Horesh et al., 2000). Why control is so important, is best reflected in the following description from a patient:

I think of my ed [eating disorder] as a sanctuary from the pain that I've lived through. I have control over myself when I restrict, and I have control over my body when I purge and that is what has got me through the hard times in my life, the times when there was no control or stability in sight. (Fox et al., 2005, p. 958)

AN is classified by the DSM-V (American Psychiatric Association, 2013) as an intense fear of gaining weight, restriction of energy intake leading to a significant low body weight, and a false experience of body weight or shape. Clinical characteristics associated with AN are negative body image, lack of self-confidence, impulsivity, perfectionism, fear of failure (performance anxiety), and self-criticism (Noordenbos \& Lammers, 2018). Many researchers and clinicians have proposed that the eating disorder is a desperate attempt to compensate for the lack of control that patients with AN experience over their lives (Bruch, 1974). It is suggested that AN patients have trouble identifying and experiencing (negative) emotions. Finding control through disordered eating behaviour functions as a strategy to cope with these negative emotions (e.g. Oldershaw et al., 2015).

Losing weight is a response to this perceived lack of control, providing a strong feeling of selfconfidence and a strong sense of self, which contributes to a more positive identity and a sense of 
control (Noordenbos, 2007, p. 46). In other words, (unhealthy) dieting behaviour leads to a feeling of being in control, which in AN patients is experienced as extremely positive. At first this behaviour might seem functional to AN patients. However, this sense of control is not permanent as AN patients have to continue and enhance these dietary behaviours, otherwise AN patients would perceive a loss of control (Fairburn et al., 1999). Over time these controlling behaviours can become habitual (Walsh, 2013), and also very harmful and dysfunctional as the patient becomes increasingly focussed on gaining control through unhealthy dieting behaviour. The paradox is that this restrictive and/or compensative behaviour to lose weight, will often take control over the patient as the disease progresses.

It takes control of you, but it can also feel very safe. It's a very confusing illness, because at the moment it's probably got a lot of control over me, in certain ways, and I just want to get away from it, I'm just sick and tired and I'm exhausted, but then it kinds of protects you as well, I think, from coping with other things... It distracts you so completely about things you don't want to think about, to lose that is quite scary. (Tan et al., 2006, p. 12)

In light of this report, it is understandable why it is so hard to recover from AN, as this feeling of control - gained through (unhealthy) dieting behaviour, provides a sense of safety which at the same time gets tied into the patient's sense of self (Noordenbos, 2007; Walsh, 2013). Losing one's eating disorder would be experienced as losing one's safety and sense of self, which is a fear invoking experience.

Some people said to me, "If I could wave a magic wand and get rid of anorexia, wouldn't you like that?" and I was, like, "Well, no, because it's, I'm, it's safe, it's what you know." And although it's killing you, it's what you know, and that's, that's my identity, having anorexia. ... I was too scared because that was who I was, ... 
because I'd be losing ME. Who would I be if I didn't, if I wasn't that? I was too scared

to make that decision to give it up. (Hope et al., 2018, p. 24)

The above section highlighted the importance of control in AN. Patients experience lack of control over their lives and attempt to regain control though disordered eating. Given the clinical relevance of this experienced lack of control, it is important to fully understand all aspects of the sense of control. Previous work has mainly focused on the self-reported experience of control in AN, however a more implicit sense of control, such as the SoA, has not yet been studied. Focusing on mechanisms of implicit SoA may help to illuminate the underlying cause of experienced loss of self-control in AN. Furthermore, adopting implicit measures can help to avoid some of the classic issues related to explicit self-report measures (e.g. demand characteristics), a much debated issue within research into AN (Engel et al., 2021).

SoA is defined by (Haggard, 2017) as the association between a voluntary action and outcome - e.g. flipping the switch (voluntary action) that turns on the light (outcome). Haggard (2017) described two key mechanisms that are necessary for the SoA to arise, ownership ('my body moved') and volition ('I voluntary made it move'). For a more detailed reading on neurological and cognitive mechanisms involved in the SoA see (Haggard, 2017; Moore \& Haggard, 2008; Moore \& Obhi, 2012; Wen \& Haggard, 2020). SoA is often measured with the intentional binding (IB) task (Haggard et al., 2002; Moore \& Obhi, 2012). This task is an adapted version of the Libet clock method (Libet et al., 1993) and measures the perceived time of action (often a key press) and a sensory outcome (e.g. auditory tone). When participants are under the impression that they cause the tone, a temporal binding occurs and participants estimate the time of action and the occurrence of the tone as closer together (Haggard, 2017).

Targeting the SoA is a useful way in which to explore an implicit component of the sense of control. Indeed, the SoA has been found to be altered in a variety of psychiatric and neurological disorders where deficits in the sense of control are also found, e.g. schizophrenia (Haggard et al., 2003; Hauser et al., 2011; Synofzik \& Voss, 2010) Alien hand syndrome (Jenkinson et al., 2015; Tetreault et 
al., 2020), obsessive compulsive disorder (Oren et al., 2019). Differences in SoA have also been found in other disorders such as Parkinson's disease (Saito et al., 2017) and autism spectrum disorder (Sperduti et al., 2014). These findings support the possibility that the lack of control that AN patients experience may be associated with a reduced SoA.

Research into the relationship between SoA and emotional states lends further support to the possibility that $\mathrm{AN}$ involves a reduced SoA. For example, research on healthy subjects has found that emotional states, such as fear and anger as well as general states of arousal, reduce the SoA (Christensen et al., 2019; Render \& Jansen, 2019). AN is associated with heightened anxiety which persists after recovery (Wagner et al., 2007), and the co-occurrence of anxiety disorders is found to be higher in AN patients compared healthy subjects (Kaye et al., 2013). A recent study also showed higher state anxiety in AN prior to an experimental task (Hasenack et al., 2021). It might be plausible that higher states of anxiety, lowers the SoA in AN given the evidence that fearful emotions and arousal reduce the SoA.

In sum, the need to feel in control is a central component of AN. AN patients tend to use dieting and restrictive/purging behaviour to feel a sense of control. This need for control is suggested to stem from a negative sense of self where patients have trouble in identifying and dealing with negative emotions. Previous studies showed that the SoA is lowered in psychiatric patient groups where the sense of control is also found to be impaired. Furthermore, previous literature on the SoA suggested that negative emotional states and arousal lower the SoA. As AN patients have higher anxiety levels compared to healthy participants, this might influence the SoA in AN. The current study aims to further explore differences in this relationship with the IB task. We investigated the SoA in a sample of AN patients, recovered AN (RAN) patients and healthy controls (HC). The RAN group is included to investigate if the SoA changes after a successful completion of treatment. We expect that AN patients have a lower SoA compared to HC. Furthermore, we expect that higher state anxiety predicts a lower SoA and, given the previous literature on AN and anxiety, we expect this to be the most prominent in the AN group. 


\section{Method}

\section{Ethics statement}

The ethics committee of the Utrecht University, Faculty of Social and Behavioural Sciences, Experimental Psychology approved the current study. The study adhered to the tenets of the Declaration of Helsinki (2013). Participants were informed about the study; they received oral and written information on the purpose and procedure. Informed consent forms were signed before taking part in the study.

\section{Participants}

A total of 84 females participated in this study: 22 AN patients, 30 RAN patients, and 29 HC. See Table 1 for demographic and clinical characteristics of the AN patients, RAN patients and HC. All AN and RAN reported diagnoses were checked with the SCID-IV, part H. RAN patients were considered recovered when they successfully completed their eating disorder treatment. Inclusion criteria for HC were no past or present eating disorder.

AN patients and RAN patients were recruited from the Leontienhuis, and treatment centre GGZ Rivierduinen Eetstoornissen Ursula, all located in the Netherlands. Leontienhuis is a nontreatment institution where help and support is provided by people recovered from an eating disorder. For more information see www.leontienhuis.nl. HC were volunteers from the Leontienhuis and undergraduate students, who were recruited from Utrecht University. Students received course credit for participation.

BMI was significantly different between groups $F(2,55)=6.057, p=.004$. Tukey pairwise comparison revealed that $\mathrm{AN}$ patients had a significant lower $\mathrm{BMI}$ compared to $\mathrm{HC}(\mathrm{p}=.009)$. A significant lower BMI was also found for RAN patients compared to $H C(p<.05)$. No significant difference was found in BMI between RAN and AN ( $p=.49)$. Results showed no difference in age between groups $F(2,78)=0.392, p=.677)$. See Table 1 . 


\section{Table 1}

Demographic and clinical characteristics

\begin{tabular}{llll}
\hline & \multicolumn{1}{c}{ HC } & \multicolumn{1}{c}{ RAN } & AN \\
\hline Age & $30.3 \pm 14.0$ & $32.5 \pm 10.6$ & $(19-63)$ \\
& $(18-55)$ & $(19-56)$ & $19.0 \pm 2.46$ \\
BMI & $\mathbf{2 2 . 0 \pm 2 . 7 2}$ & $20.2 \pm 2.42$ & $(14.0-22.7)$ \\
& $(18.0-28.3)$ & $(14.9-25.4)$ & $5.85 \pm 3.69$ \\
IIIness duration in & -- & $7.21 \pm 5.00$ & $(2-15)$ \\
Years & & $(2-20)$ & -- \\
Time since completion of & -- & $5.68 \pm 6.75$ & $3.27 \pm 0.52$ \\
treatment in years & & $(0-28)$ & $59.5 \pm 10.9$ \\
Self-Control Scale & $3.52 \pm 0.42$ & $3.53 \pm 0.47$ & \\
STAl-S & $35.9 \pm 8.26$ & $48.1 \pm 13.1$ & \\
\hline
\end{tabular}

\section{Materials and procedure}

After providing signed informed consent, participants took part in the interview, filled out the questionnaires and participated in the IB task in the order as presented below. Gorilla Experiment Builder (www.gorilla.sc) was used to create and host the questionnaires (Anwyl-Irvine et al., 2020).

\section{Structured and Clinical Interview for DSM (SCID) IV-I part H}

Part $\mathrm{H}$ (Eating Disorders) of the SCID IV-I interview was used to check the diagnostic criteria for the presence of an acute or past eating disorder diagnoses in participants.

\section{Self-Control Scale}


The Self-Control Scale (SCS) is a trait measure of self-control (Tangney et al., 2004). It consists of 36 statements that are scored on a 5-point scale ranging from 1 "not at all" to 5 "very much" from which a total score is calculated. The questionnaire was used for a demographic description of the sample.

\section{State-Trait Anxiety Scale}

The State-Trait Anxiety Scale (STAI) measures state and trait anxiety (Spielberger et al., 1970). The STAI consists of 40 statements that are scored on a 4 point scale ranging from 1 "almost never" to 4 "almost always". The first 20 items evaluate state anxiety and the other 20 evaluate trait anxiety. For this study only the state version (STAI-S) was administered. The sum of the first 20 items was calculated.

\section{Intentional Binding task}

An adapted version of the intentional binding task designed by Haggard, Clark and Kalogeras (2002) was used in this study. A 13-inch screen was used where the stimuli followed by an answering box were presented in a fixed order, they were both presented on a white background. The stimuli consisted of a clock, centred on the screen with rotating clock hand, see figure 1 . The hand rotated with a speed of $2560 \mathrm{~ms}$. The starting location of the dot was determined randomly for every trial. The clock hand stopped rotating between 1500 ms and 2500 after a keypress (spacebar) or auditory tone $(1,000 \mathrm{~Hz}, 100 \mathrm{~ms}$ duration). The answering screen appeared with a question to report the time of the keypress and tone and an answering box which only logged numbers between 0-60.

\section{Figure 1}

Schematic overview of clock presented during experiment, with clockwise rotating hand.

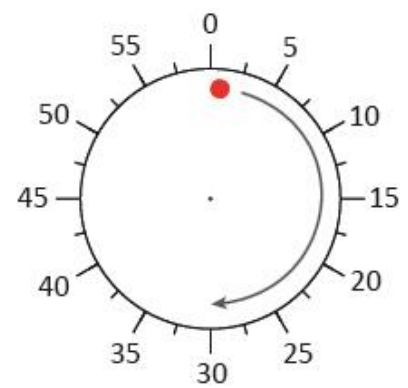


In the IB task, participants judged the subjective time of a simple voluntary action (keypress) or an auditory tone. Participants were asked to sit approximately $30 \mathrm{~cm}$ from the screen and fixate on the fixation dot in the centre, while the clock hand rotated until the trial was completed. The task consisted of 4 conditions ( 2 baseline, and 2 agency conditions) with 28 trials in each. The first 3 trials were considered practice trials, the last 25 trials were used for analysis. Note that the trials were shortened for this study, in the original 40 trials per condition are used. This was done to keep the experiment short due to the low attention span of AN patients.

In the Baseline Tone condition, participants were presented with an auditory tone and were asked to judge the time they heard the tone. In the Baseline Action condition, participants were asked to press the spacebar at a moment of their choosing and were asked to judge the time they pressed the spacebar (action judgement). In the two agency conditions the participants were asked to press the spacebar, which was followed by the auditory tone. Participants were asked to make a judgement of the time of the tone (Agency Tone) and a judgement of the time of action (Agency Action). In the agency conditions, the auditory tone was presented with a fixed temporal window, 250 ms after the spacebar was pressed. The conditions were randomly presented for each participant. See figure 2 for an overview of conditions. 
Figure 2

IB task with Baseline and Agency conditions

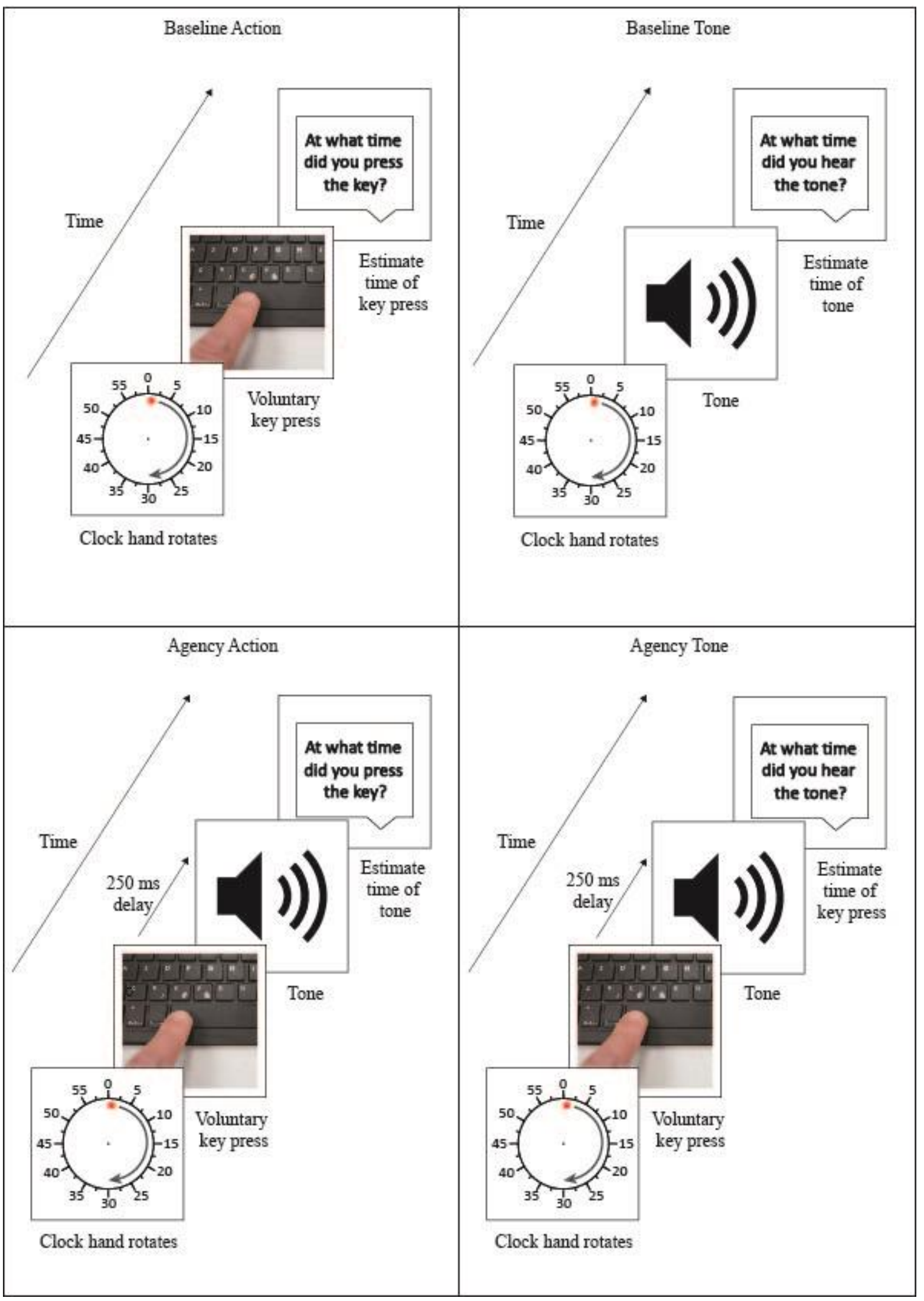




\section{Statistical analysis}

All data were analysed in $R$ (v3.6.3; R Core team, 2020). The STAI-S scores were derived by summing item scores.

For the IB task, the variable of interest were the timing errors in judgement for each condition. To calculate the IB, the judgement errors were transformed into milliseconds by dividing the rotation time (which is $2560 \mathrm{~ms}$ ) by 60 . Next the action-shift, tone-shift and IB were calculated with the following formulas:

$$
\begin{aligned}
& \text { action-shift } \text { Agency }_{\text {Action }}-\text { Baseline }_{\text {Action }} \\
& \text { tone-shift }=\text { Baseline }_{\text {Tone }}-\text { Agency }_{\text {Tone }} \\
& \text { IB }=\text { action-shift }+ \text { tone-shift }
\end{aligned}
$$

A higher value reflects a stronger IB.

An one-way ANOVA was used to calculate differences between Group (AN, RAN, HC) for the STAI-S scores. A mixed ANOVA was used to analyse differences in judgement errors with Group (AN, RAN, HC) as between variable and Condition (Baseline, Agency) and Event (Action, Tone) as within variables. Planned comparisons were Tukey corrected. Three regression analyses were conducted with IB scores, action-shift and tone-shift as the dependent variables and STAI-S scores and Group as independent variables.

The assumption of normality was assessed with the Shapiro-Wilks test and data plots. Homogeneity of variance was assessed with the Levine's test.

We checked for correlations of all agency measurements with, 'age', 'BMI', 'SCS', 'duration ED', 'duration ED treatment', and 'time since completion ED treatment', as described in Table 1. There were some significant correlations, but they did not survive the Bonferroni correction (critical $p=.007$; all $\left.p^{\prime} s>.035\right)$ and were therefore not used as covariates in analyses. 


\section{Results}

\section{STAI-S}

An one-way ANOVA showed significant between Group differences for state anxiety, $F(2,79)=29.4, p$ $<.001$. AN patients showed higher STAI-S scores compared to RAN patients $(p=.001)$ and HC $(p<.001)$. RAN patients have higher STAI-S scores compared to HC $(\mathbf{p}<.001)$, see Table 1 and figure 3.

\section{Figure 3}

Mean STAI-State scores of Group.

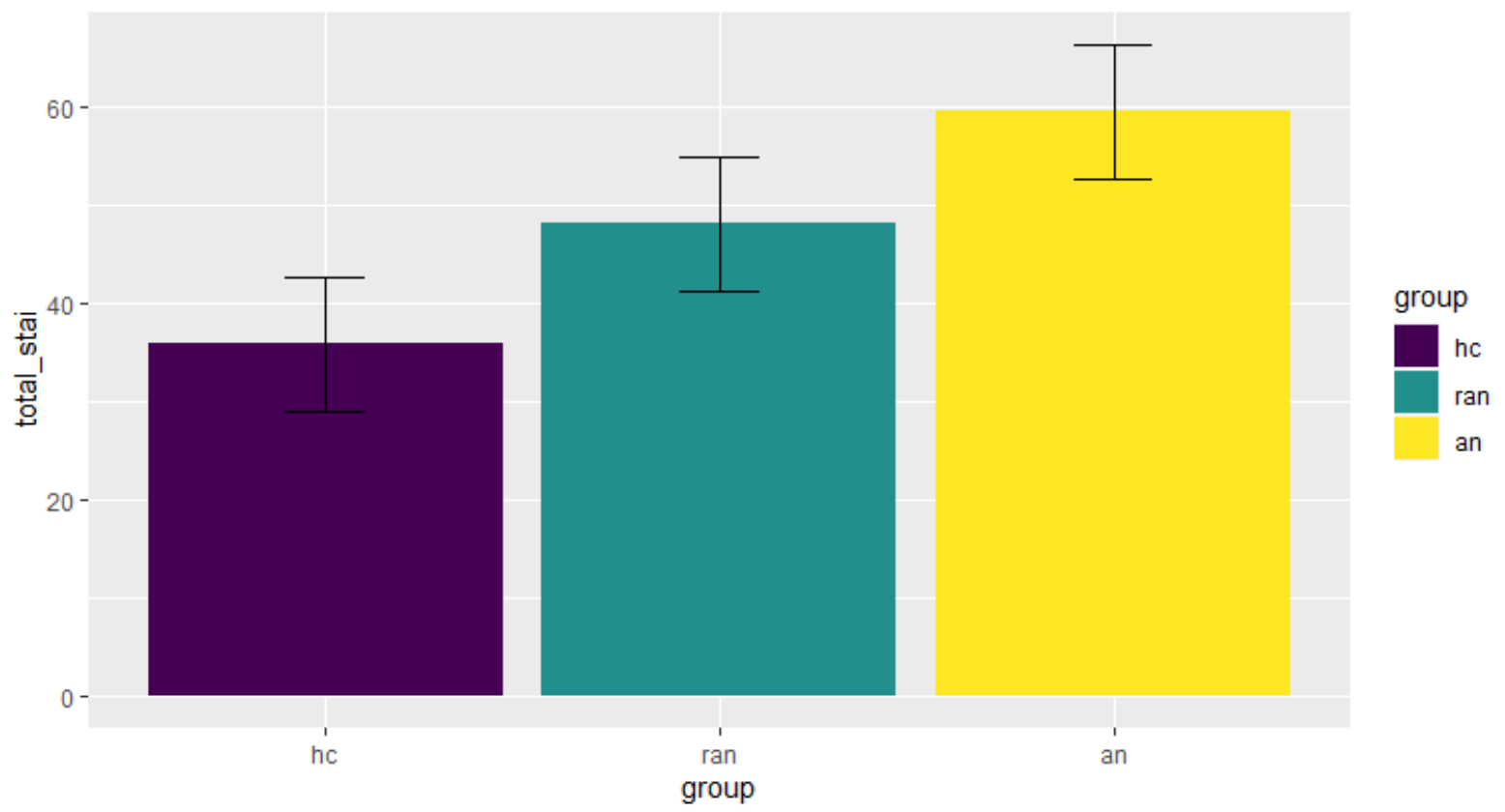

Note. Errorbars depict SE.

IB

The results of the mixed ANOVA revealed a significant main effect of Event and a significant interaction effect of Condition by Event. This interaction effect indicates a temporal binding in the Agency Conditions, see figure 4. In contrast to our expectations, we did not find a main effect of Group. See Table 2 for statistics and figure 4 for means in judgement error per Condition. 


\section{Table 2}

Results of mixed ANOVA

\begin{tabular}{lccc}
\hline \multicolumn{1}{c}{ Effect } & $D f$ & $F$ & $p$ \\
\hline Group & 2,78 & 0.238 & 0.79 \\
Event & $\mathbf{1 , 7 8}$ & $\mathbf{5 0 . 2 3 5}$ & $<.001$ \\
Condition & 2,78 & 2.508 & .12 \\
Group:Event & 2,78 & 2.028 & .14 \\
Group:Condition & 2,78 & 1.405 & .25 \\
Event:Condition & $\mathbf{1 , 7 8}$ & $\mathbf{1 1 5 . 0 5 7}$ & $<.001$ \\
Group:Event:Condition & 2,78 & 2.385 & .10 \\
\hline
\end{tabular}

\section{Figure 4}

Means in judgement errors for Baseline Action and Agency Action (left) and Baseline Tone and Agency Tone (right)

Mixed Anova, $F(1,78)=115.06, p=<0.0001, \eta_{g}^{2}=0.17$

$$
\text { group 追 hc 它 ran 户 an }
$$

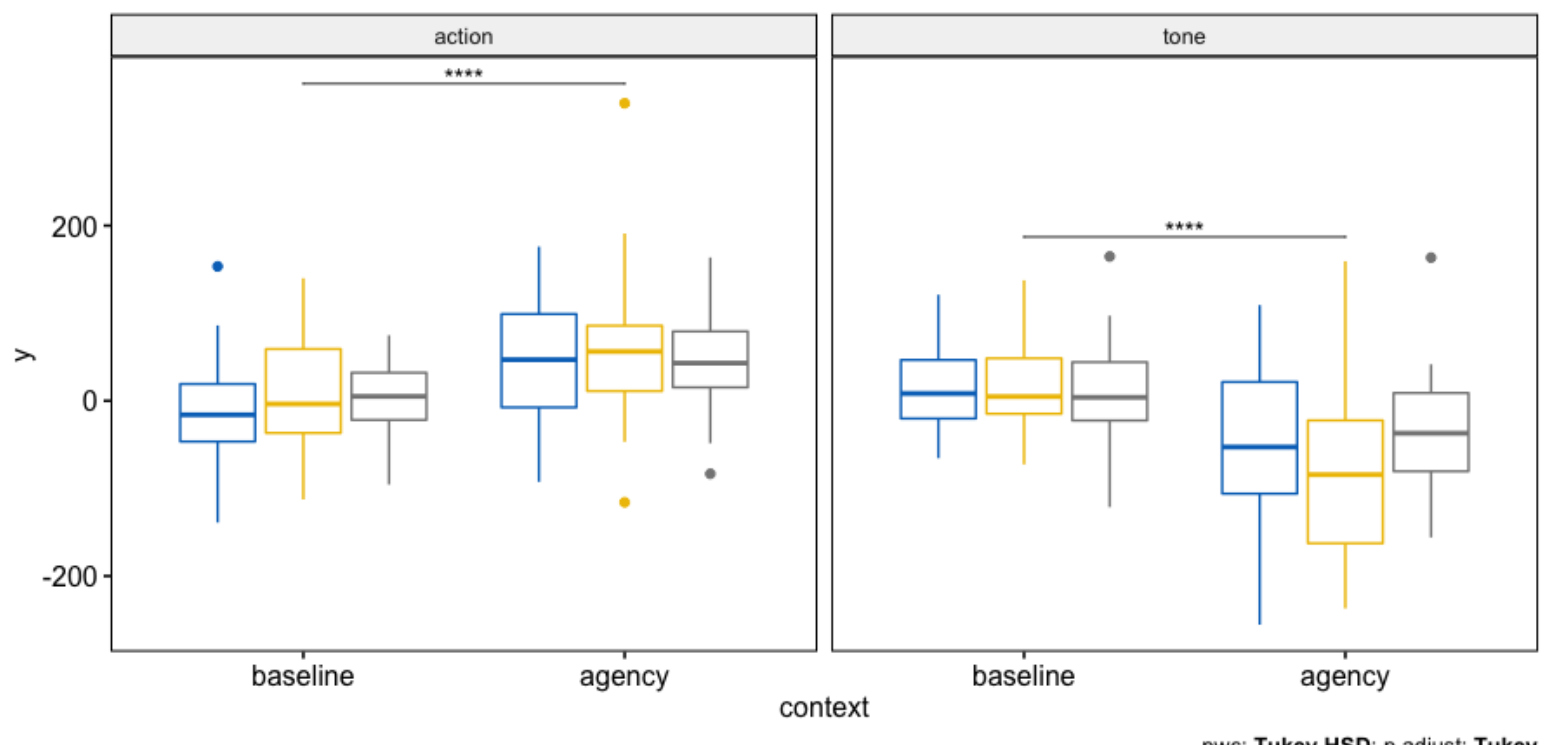




\section{STAI-S and IB}

In contrast to expectations, the results of the regression indicated that STAI-S scores did not significantly predict IB scores $\left(R^{2}=.01, F(5,75)=1.09, p=.37\right)$, nor action-shift $\left(R^{2}=-0.03, F(5,75)=\right.$ $0.62, p=0.69)$ or tone-shift $\left(R^{2}=.02, F(5,75)=1.28, p=.28\right)$. See Tables 3,4 and 5 for the model summaries.

Table 3

Model summary IB

\begin{tabular}{lcccc}
\hline & Beta & Std. Error & $t$ & $p$ \\
\hline Intercept & 177.72 & 90.60 & 1.96 & .05 \\
STAI-S & -1.24 & 2.46 & -0.50 & .62 \\
RAN & -20.08 & 117.55 & -0.17 & .86 \\
AN & 12.54 & 158.81 & 0.08 & .94 \\
STAI-S:RAN & 1.23 & 2.89 & 0.43 & .67 \\
STAI-S:AN & -0.41 & 3.27 & -0.12 & .90 \\
\hline
\end{tabular}

Table 4

Model summary Action Shift

\begin{tabular}{lcccc}
\hline & Beta & Std. Error & $t$ & $p$ \\
\hline Intercept & 41.50 & 49.53 & 0.84 & .41 \\
STAI-S & 0.57 & 1.35 & 0.43 & .67 \\
RAN & 59.29 & 64.26 & 0.92 & .36 \\
AN & 59.18 & 86.82 & 0.68 & .50 \\
STAI-S:RAN & -1.82 & 1.58 & -1.00 & .34 \\
STAI-S:AN & -1.50 & 1.79 & -0.84 & .41 \\
\hline
\end{tabular}


Table 5

Model summary Tone Shift

\begin{tabular}{lcccc}
\hline & Beta & Std. Error & $t$ & $p$ \\
\hline Intercept & 136.23 & 75.63 & 1.80 & .08 \\
STAI-S & -1.81 & 2.06 & -0.88 & .38 \\
RAN & -79.37 & 98.13 & -0.81 & .42 \\
AN & -46.65 & 132.58 & -0.35 & .73 \\
STAI-S:RAN & 2.74 & 2.41 & 1.14 & .26 \\
STAI-S:AN & 1.09 & 2.73 & 0.40 & .69 \\
\hline
\end{tabular}

\section{Discussion}

The need to feel control is a central component in AN. Previous studies have suggested that the disordered eating behaviour functions as a strategy to feel in control over difficult situations, experiences, and emotions (Oldershaw et al., 2015). This coping behaviour creates a temporary, albeit very positive and rewarding, feeling of being in control. It is this reinforcing aspect, that makes AN especially hard to treat, and that can be seen as a maintaining factor of the disorder. Since control appears to be such an essential factor in development and maintenance of the disorder, it is important to fully understand all aspects of control in AN. One of those aspects is the SoA, a more implicit sense of control which has not yet been studied in AN. Here we investigated if AN patients have a lower SoA compared to HC. Furthermore, we investigated whether anxiety predicts SoA in AN, as AN patients are known to have higher anxiety (Hasenack et al., 2021), and previous studies have found that negative affect lowers the SoA in healthy subjects (Christensen et al., 2019).

Results of the intentional binding task showed a temporal binding for all participants: In the agency conditions, there was an action-shift towards the tone and a tone-shift towards the action. 
These findings are in line with previous results on the IB task (Moore \& Obhi, 2012). In contrast to expectations, our results did not show any group differences on the IB task, indicating that SoA is not different between AN and RAN patients and HC. Interestingly, state anxiety was not a significant predictor for the IB. In other words, in contrast to our expectations, we did not find any evidence that state anxiety influences the SoA.

One reason for why this study found no difference in IB scores between groups might simply be that the specific mechanisms underlying the IB task are not impaired or altered in AN patients (in contrast to schizophrenic patients, for example, who show an enhanced retrospective binding (Synofzik \& Voss, 2010; Voss et al., 2010). Consequently, the deficit in control associated with AN may be best conceptualised in terms of negative attitudes towards themselves and their ambitions - for example, "despair over defining or becoming the person they want to be" - (Bers et al., 2004) rather than the feeling of control over action and sensory outcome, which is targeted by the IB. Instead, dieting as a means to gain control, may be triggered by negative affect and serve as a method to copeor avoid emotional distress (Noordenbos, 2007).

Nevertheless, agency is a complex construct and the IB task only measures binding over an action and fixed sensory outcome and is a relatively predictable task. Therefore, it might only tap into some aspects of agency, and our findings might not necessarily generalise to all aspects of agency. For example, a recent study of Perrykkad et al (2021) investigated the predictive processing account of agency, which situates agency in a broader scheme of action and policy selection. Here the authors incorporate different degrees of uncertainty and showed that uncertainty over actions negatively influenced the SoA (for a detailed overview of this study see Perrykkad et al., 2021). Such paradigms, that modulate uncertainty might be more informative of the SoA in AN, as AN patients experience higher anxiety and higher need for control in uncertain situations (Sternheim et al., 2011).

This study did not find an influence of state-anxiety on the SoA. Previous studies have found that general states of arousal as well as negative emotional states, such as fear and anger, reduce the SoA in the IB task (Christensen et al., 2019; Render \& Jansen, 2020). Although anxiety and fear share 
the same underlying brain and behavioural mechanisms, they are two distinct processes; fear is a directional state while anxiety is more diffuse (Barlow, 2004). Therefore fear - as opposed to anxiety - might have an influence on the temporal binding. For example, Christensen et al. (2019), used painful shocks during some trials of the IB, where they manipulated their paradigm in such a way that fear was directly related to the task. Our study did not specifically manipulate emotional states, but only measured the level of state-anxiety between groups before the experiment. However, further studies on anxiety and SoA are needed to the precise relation between fear, anxiety and SoA.

In conclusion, the aim of this study was to investigate an implicit sense of control (SoA) through an objective measure (IB task). We did not find any evidence of altered SoA, as assessed by the IB task, in AN patients. We also did not find state anxiety to be a predictor for the SoA. Further studies should investigate other features of the SoA that are related to aspects where AN experience less control over, by for example manipulating uncertainty.

\section{References}

American Psychiatric Association. (2013). Diagnostic and Statistical Manual of Mental Disorders (5th ed.). American Psychiatric Publishing.

Anwyl-Irvine, A. L., Massonnié, J., Flitton, A., Kirkham, N., \& Evershed, J. K. (2020). Gorilla in our midst: An online behavioral experiment builder. Behavior Research Methods, 52(1), 388-407. https://doi.org/10.3758/s13428-019-01237-x

Barlow, D. H. (2004). Anxiety and its disorders: The nature and treatment of anxiety and panic (Second Edition). The Guilford Press.

Bers, S. A., Blatt, S. J., \& Dolinsky, A. (2004). The sense of self in anorexia-nervosa patients: A psychoanalytically informed method for studying self-representation. In Psychoanalytic Study of the Child (Vol. 59, pp. 294-315). Yale University Press.

https://doi.org/10.1080/00797308.2004.11800742 
Bruch, H. (1974). Eating disorders. Obesity, anorexia nervosa, and the person within. Routledge \& Kegan Paul. https://www.cabdirect.org/cabdirect/abstract/19741422333

Christensen, J. F., di Costa, S., Beck, B., \& Haggard, P. (2019). I just lost it! Fear and anger reduce the sense of agency: a study using intentional binding. Experimental Brain Research, 237(5), 12051212. https://doi.org/10.1007/s00221-018-5461-6

Engel, M. M., Gadsby, S., Corcoran, A. W., Keizer, A., Dijkerman, H. C., \& Hohwy, J. (2021). Waiting longer, feeling fatter: Effects of response delay on tactile distance estimation and confidence in females with anorexia nervosa. https://doi.org/https://doi.org/10.31234/osf.io/gvr2h

Fairburn, C. G., Cooper, Z., \& Shafran, R. (2003). Cognitive behaviour therapy for eating disorders: A "transdiagnostic" theory and treatment. In Behaviour Research and Therapy. https://doi.org/10.1016/S0005-7967(02)00088-8

Fairburn, C. G., Shafran, R., \& Cooper, Z. (1999). A cognitive behavioural theory of anorexia nervosa. Behaviour Research and Therapy, 37(1), 1-13. https://doi.org/10.1016/S0005-7967(98)00102-8

Fox, N., Ward, K., \& O'Rourke, A. (2005). Pro-anorexia, weight-loss drugs and the internet: an "antirecovery" explanatory model of anorexia. Sociology of Health and IIIness, 27(7), 944-971. https://doi.org/10.1111/j.1467-9566.2005.00465.x

Froreich, F. v., Vartanian, L. R., Grisham, J. R., \& Touyz, S. W. (2016). Dimensions of control and their relation to disordered eating behaviours and obsessive-compulsive symptoms. Journal of Eating Disorders, 4(1). https://doi.org/10.1186/s40337-016-0104-4

Haggard, P. (2017). Sense of agency in the human brain. Nature Reviews Neuroscience, 18, 197-208.

Haggard, P., Clark, S., \& Kalogeras, J. (2002). Voluntary action and conscious awareness. Nature Neuroscience, 5(4), 382-385. https://doi.org/10.1038/nn827 
Haggard, P., Martin, F., Taylor-Clarke, M., Jeannerod, M., \& Franck, N. (2003). Awareness of action in schizophrenia. NeuroReport, 14(7), 1081-1085.

https://doi.org/10.1097/01.wnr.0000073684.00308.c0

Hasenack, B., Sternheim, L., Bijsterbosch, J., \& Keizer, A. (2021). The link between anxiety and assessment of body attitudes and body size estimation in anorexia nervosa. Journal of Experimental Psychopathology, 1-8.

Hauser, M., Moore, J. W., de Millas, W., Gallinat, J., Heinz, A., Haggard, P., \& Voss, M. (2011). Sense of agency is altered in patients with a putative psychotic prodrome. Schizophrenia Research, 126(1-3), 20-27. https://doi.org/10.1016/j.schres.2010.10.031

Hope, N. H., Wakefield, M. A., Northey, L., \& Chapman, A. L. (2018). The association between locus of control, emotion regulation and borderline personality disorder features. Personality and Mental Health, 12(3), 241-251. https://doi.org/10.1002/pmh.1419

Horesh, N., Zalsman, G., \& Apter, A. (2000). Internalized anger, self-control, and mastery experience in inpatient anorexic adolescents. Journal of Psychosomatic Research, 49(4), 247-253.

Jenkinson, P. M., Edelstyn, N. M. J., Preston, C., \& Ellis, S. J. (2015). Anarchic hand with abnormal agency following right inferior parietal lobe damage: a case report. Neurocase, 21(4), 471-478. https://doi.org/10.1080/13554794.2014.925936

Kaye, W. H., Wierenga, C. E., Bailer, U. F., Simmons, A. N., \& Bischoff-grethe, A. (2013). Nothing tastes as good as skinny feels : the neurobiology of anorexia nervosa. Trends in Neurosciences, 36(2), 110-120. https://doi.org/10.1016/j.tins.2013.01.003

Libet, B., Libet, B., Gleason, C. A., Wright, E. W., \& Pearl, D. K. (1993). Time of Conscious Intention to Act in Relation to Onset of Cerebral Activity (Readiness-Potential). In Neurophysiology of 
Consciousness (pp. 249-268). Birkhäuser Boston. https://doi.org/10.1007/978-1-4612-03551_15

Moore, J. W., \& Haggard, P. (2008). Awareness of action: Inference and prediction. Consciousness and Cognition, 17(1), 136-144. https://doi.org/10.1016/j.concog.2006.12.004

Moore, J. W., \& Obhi, S. S. (2012). Intentional binding and the sense of agency: A review. Consciousness and Cognition, 21(1), 546-561. https://doi.org/10.1016/j.concog.2011.12.002

Noordenbos, G. (2007). Gids voor herstel van eetstoornissen. De Tijdstroom.

Noordenbos, G., \& Lammers, M. (2018). Psychische achtergronden. In G. Noordenbos \& A. van Elburg (Eds.), Handboek Eetstoornissen (3rd ed., pp. 99-108). De Tijdsstroom Uitgeverij.

Oldershaw, A., Lavender, T., Sallis, H., Stahl, D., \& Schmidt, U. (2015). Emotion generation and regulation in anorexia nervosa: A systematic review and meta-analysis of self-report data. Clinical Psychology Review, 39, 83-95. https://doi.org/10.1016/j.cpr.2015.04.005

Oren, E., Eitam, B., \& Dar, R. (2019). Intentional binding and obsessive-compulsive tendencies: A dissociation between indirect and direct measures of the sense of agency. Journal of ObsessiveCompulsive and Related Disorders, 20, 59-65. https://doi.org/10.1016/j.jocrd.2017.11.002

Render, A., \& Jansen, P. (2019). Dopamine and sense of agency: Determinants in personality and substance use. PLOS ONE, 14(3). https://doi.org/10.1371/journal.pone.0214069

Render, A., \& Jansen, P. (2020). Influence of arousal on intentional binding: Impaired action binding, intact outcome binding. Attention, Perception, and Psychophysics. https://doi.org/10.3758/s13414-020-02105-z

Saito, N., Takahata, K., Yamakado, H., Sawamoto, N., Saito, S., Takahashi, R., Murai, T., \& Takahashi, H. (2017). Altered awareness of action in Parkinson's disease: Evaluations by explicit and implicit measures. Scientific Reports, 7(1). https://doi.org/10.1038/s41598-017-08482-0 
Sperduti, M., Pieron, M., Leboyer, M., \& Zalla, T. (2014). Altered pre-reflective sense of agency in autism spectrum disorders as revealed by reduced intentional binding. Journal of Autism and Developmental Disorders, 44(2), 343-352. https://doi.org/10.1007/s10803-013-1891-y

Spielberger, C. D., Gorsuch, R. L., \& Lushene, R. E. (1970). State-trait anxiety inventory (test manual). Consulting Psychologists Press.

Sternheim, L., Startup, H., \& Schmidt, U. (2011). An experimental exploration of behavioral and cognitive-emotional aspects of intolerance of uncertainty in eating disorder patients. Journal of Anxiety Disorders. https://doi.org/10.1016/j.janxdis.2011.03.020

Synofzik, M., \& Voss, M. (2010). Disturbances of the sense of agency in schizophrenia. In Neuropsychology of the Sense of Agency: From Consciousness to Action (pp. 145-155). Springer Milan. https://doi.org/10.1007/978-88-470-1587-6_8

Tan, J. O. A., Hope, T., Stewart, A., \& Fitzpatrick, R. (2006). Competence to make treatment decisions in anorexia nervosa: thinking processes and values. Philos Psychiatr Psychol, 13(4), 267-282. https://doi.org/10.1038/mp.2011.182.doi

Tangney, J. P., Baumeister, R. F., \& Boone, A. L. (2004). High self-control predicts good adjustment, less pathology, better grades, and interpersonal success. J Pers, 72(2), 271-324. https://doi.org/http://dx.doi.org/10.1111/j.0022-3506.2004.00263.x.

Tetreault, A. M., Phan, T., Petersen, K. J., Claassen, D. O., Neth, B. J., Graff-Radford, J., Albrecht, F., Fliessbach, K., Schneider, A., Synofzik, M., Diehl-Schmid, J., Otto, M., Schroeter, M. L., \& Darby, R. R. (2020). Network Localization of Alien Limb in Patients with Corticobasal Syndrome. Annals of Neurology, 88(6), 1118-1131. https://doi.org/10.1002/ana.25901 
Voss, M., Moore, J., Hauser, M., Gallinat, J., Heinz, A., \& Haggard, P. (2010). Altered awareness of action in schizophrenia: A specific deficit in predicting action consequences. Brain. https://doi.org/10.1093/brain/awq152

Wagner, A., Aizenstein, H., Venkatraman, V. K., Julie Fudge, M., Christopher May, J., Laura Mazurkewicz, B., Guido Frank, B. K., Bailer, U. F., Fischer, L., van Nguyen, B., Carter, C., Putnam, K., \& Walter Kaye, M. H. (2007). Article Altered Reward Processing in Women Recovered From Anorexia Nervosa. In Am J Psychiatry (Vol. 164).

Walsh, B. T. (2013). Reviews and Overviews The Enigmatic Persistence of Anorexia Nervosa. American Journal of Psychiatry , 170, 477-484.

Wen, W., \& Haggard, P. (2020). Prediction error and regularity detection underlie two dissociable mechanisms for computing the sense of agency. Cognition, 195. https://doi.org/10.1016/j.cognition.2019.104074 